\title{
A Novel Approach Proposed for Human Recognition using GAIT
}

\author{
Zankhana Purohit \\ CE Department \\ Parul Institute of Engineering \& Technology
}

\author{
Mukesh Sakle \\ IT Department \\ Parul Institute of Engineering \& Technology
}

\begin{abstract}
Gait recognition is used to recognize the human by his/her walking styles. It is used in biometric authentication system, the gait is the walking sequence of human and another type of spices. In the previous methodologies a change of viewing angle causes a significant challenge for gait recognition. Proposed methodology, formulate a framework to estimate the view angle of each probe gait sequence. In this method, each gait image is represented as a set of dual-tree complex wavelet transform (DTCWT) features derived from different scales and orientations together with the $x-y$ coordinates. The proposed representation technique is capable of capturing variations. Features extraction is done using Hough Transform. Then using the similarity of features we can recognize the person details. For classification, SVM classifier is used.
\end{abstract}

\section{Keywords}

DTCWT; SVM; Hough Transform; Interval valued Feature; Fuzzy Logic.

\section{INTRODUCTION}

Gait Recognition is one of the biometric methods which has developed a current concentration in computer vision. Gait Recognition is a job to identify or authenticate the individuals through the way they walk ${ }^{[1]}$. The capability to recognize persons at a distance with the help of a camera is a desired property and is gradually vital for surveillance and other applications ${ }^{[2]}$. Gait as a biometric source, can be attained at a distance without the cooperation of person who is walking and even awareness /knowledge of the person is not important or even not required for some quick cautions or monitoring applications that require to perform recognition when the person is far away ${ }^{[3,4]}$. There is a necessity of real-world system which can support the authentication and Verification process by means of which one has to go through in order to access highly protected data in various areas ${ }^{[5]}$. Gait can be used in situations when other biometric characteristics for instance face, iris and finger print evidence do not have adequate perseverance required for recognition. Numerous researchers have made efforts to come out with proficient and stout techniques to arrest and describe human gait.

\section{EXISTING WORK}

The existing method of gait recognition comprises of three main segments i.e., feature extraction, representation and similarity computation for recognition of various activities viz., different walking styles for instance Normal walk, Moon walk, Limb walk etc. A gait cycle is an elementary unit of gait and it refers to the time interlude amid consecutive instances of initial foot-to- floor contact for the same foot ${ }^{[6]}$.
The input to the existing methodology is a video in avi format. The gait recognition systems projected in the collected works ${ }^{[7]}$ have supposed that the speed in any specific gait series is persistent. In the existing work this assumption is also considered since length of all occurrences/cases (viz., change in viewing angle, change in cloth type, carrying a bag, change in walking surface) of a person/subject is persistent. Conversely, speed could fluctuate between reference and test/probe series. Subsequently as the gait recognition count on the shape of the body outline deviations with respect to time, the variations are bagged through extracting features by keeping the axis of least inertia of a silhouette as a locus. The axis of least inertia is calculated ${ }^{[8,9]}$ for each silhouette in a gait cycle. The axis is found to be distinctive to a specific silhouette and so consequently it is used in order to extract features from a gait silhouette (Gait is extracted).

Hauling out / extracting related features for portraying a gait is an essential step in any gait recognition system. Fig 1 depicts an instance of silhouette with axis of least inertia as a locus line and the points considered for feature extraction. The features selected are : distance amid centroid C and E1 (d1), distance among centroid C and E2 (d2), parallel distance amid left border point $\mathrm{L}$ on the silhouette and centroid $\mathrm{C}(\mathrm{d} 3)$, parallel distance amongst right margin point $\mathrm{R}$ on the silhouette and centroid C (d4) and angle of axis of least inertia $(\theta)^{[10]}$. The features enumerated overhead are extracted for each th silhouette in a gait cycle. The gait of an individual may fluctuate to some extent as a result of change in clothes, walking surface, or because of carrying conditions. Such distinctions can commendably be controlled by amalgamating the features in the form of an interval type data. Hence, the features conversed above are extracted from the gait of a person of all occurrences (change in clothes, due to carrying conditions and different normal walking conditions) and the features relates to a particular silhouette of all occurrences are combined to form an interval. Therefore the gait data of a specific person is efficiently denoted/represented as intervalvalued type feature vector in the knowledge-base, which is used at the time of gait recognition. At last similarity computation is done for recognition of person. The person is recognized and Recognition rate and elapsed time is displayed. 


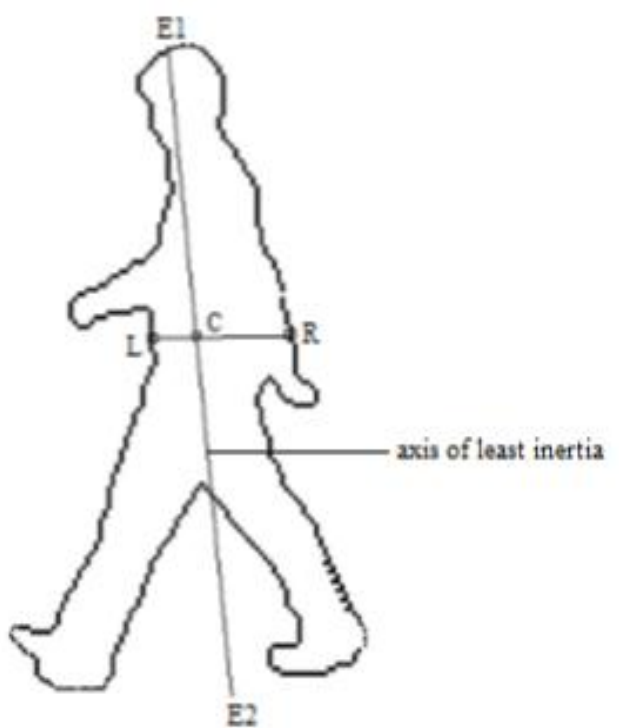

Fig. 1. Features

\section{PROPOSED APPROACH}

Proposed scheme is a symbolic approach to human gait recognition. This method is capable of capturing sequence in gait due to change in objects such as in cloth, carrying a bag and different instances of normal walking conditions more effectively. The proposed method estimate the view angle of each probe gait sequence. Each gait image is represented as a set of dual-tree complex wavelet transform (DTCWT) features derived from different scales and orientations together with the $\mathrm{x}-\mathrm{y}$ coordinates. The proposed representation technique is capable of capturing variations. Then using the similarity of features we can recognize the person details. SVM classifier is used for classification.

In the proposed methodology Recognition Rate is the parameter which is to be improved. In the proposed method the gait image features are extracted based on the parameters like Scale and Orientation together with the $x-y$ coordinates (Positions). First of all standard CASIA dataset-B is used for the implementation. Then training set ans testing set is created. In training set data of three persons is taken for different angles viz., 0,45 and 90. There are 3 test datasets in each dataset only 20 images from the total images of training set are taken. Then we can select any of the dataset and walking sequence of selected dataset is presented. Then Background subtraction of the image sequences is done and the person is tracked using Bounding Box. Then after Gait is extracted using Morphological Skeletization. Hough Transform is used to extract the features and the graph is generated and the values generated in the hough transform graph are stored in the mat file and that values are fed into SVM classification. The Graphs are generated for SVM classification and SVM testmoments are used for Recognition of the person. At, last person is recognized and Recognition Rate as well as elapsed time is displayed. The average Recognition Rate of the Proposed system is found to be more then the existing one.

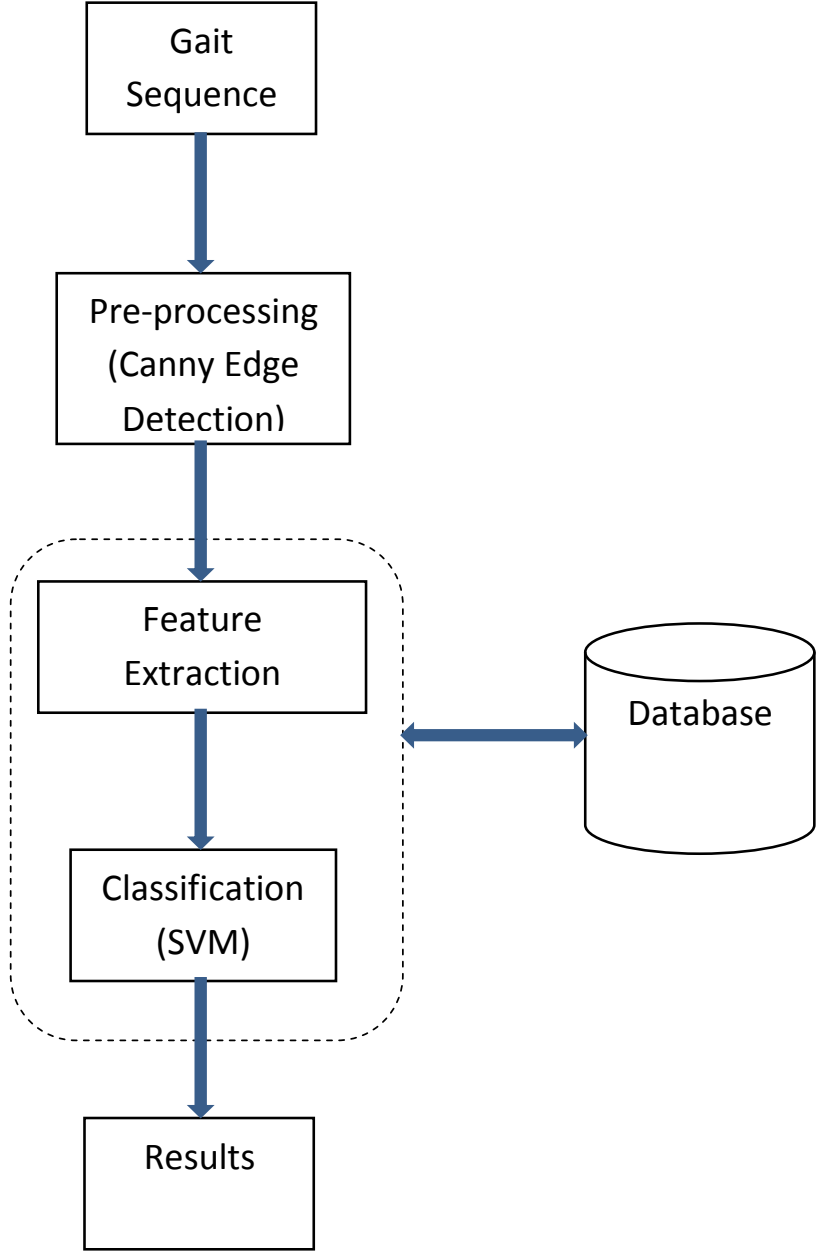

Fig. 2. Proposed Flow

\section{RESULTS OF PROPOSED WORK}

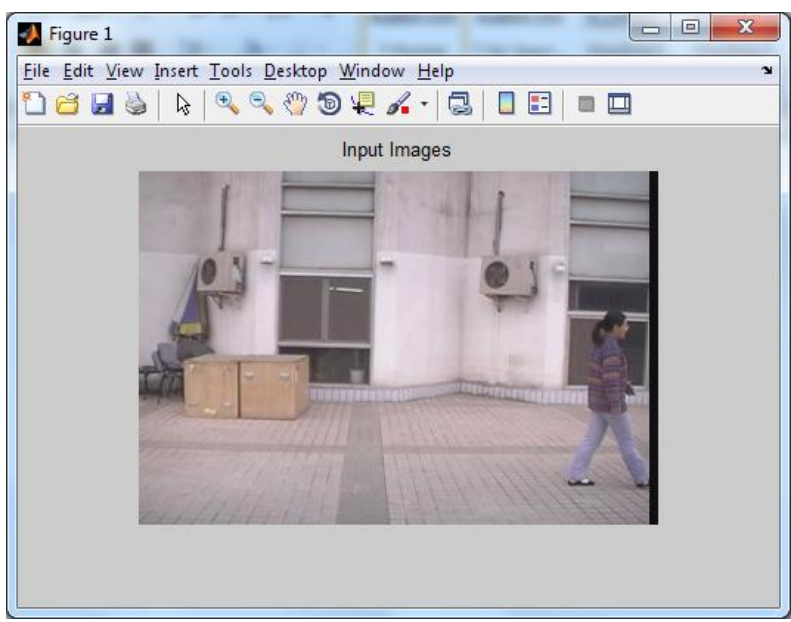

Fig. 3. Selected Image sequence 


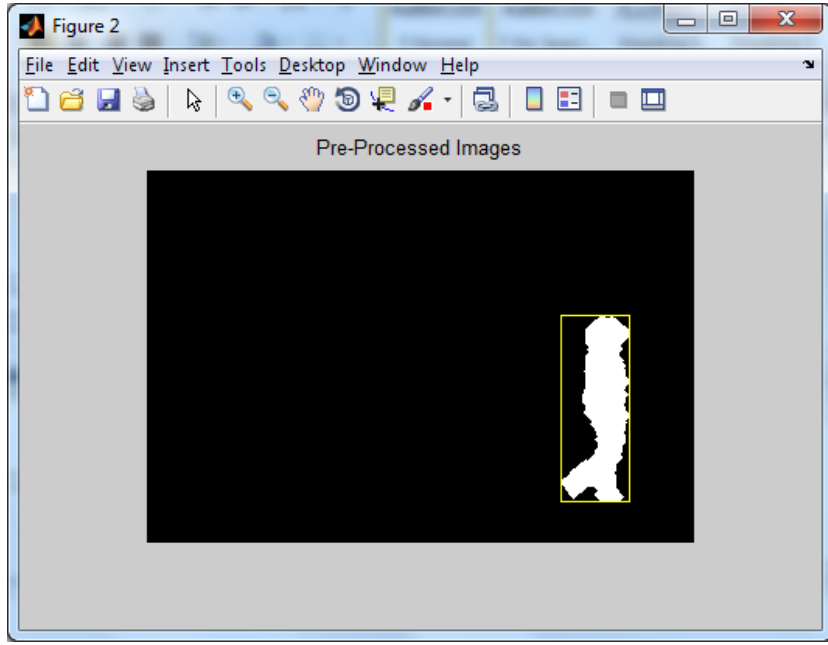

Fig. 4. Background Subtraction and Person Tracking

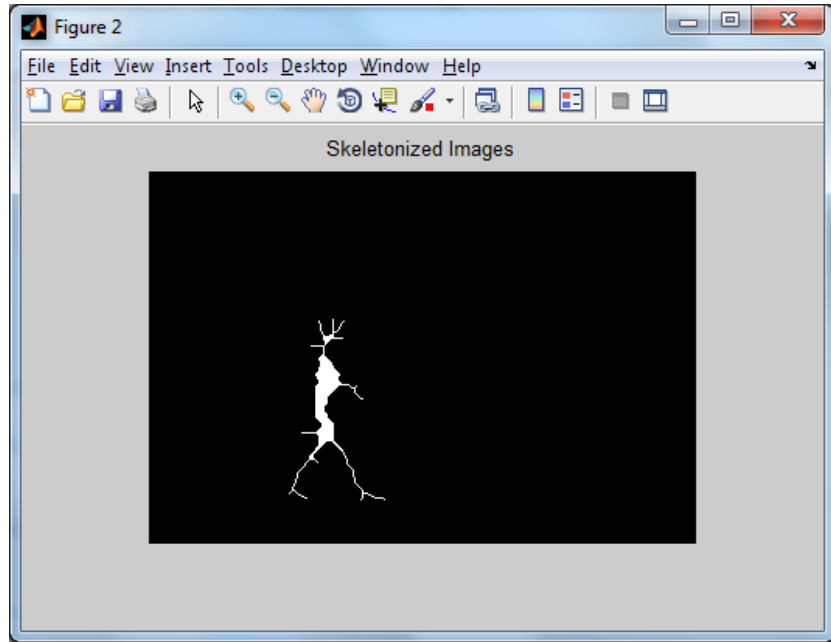

Fig.5. Gait Extraction

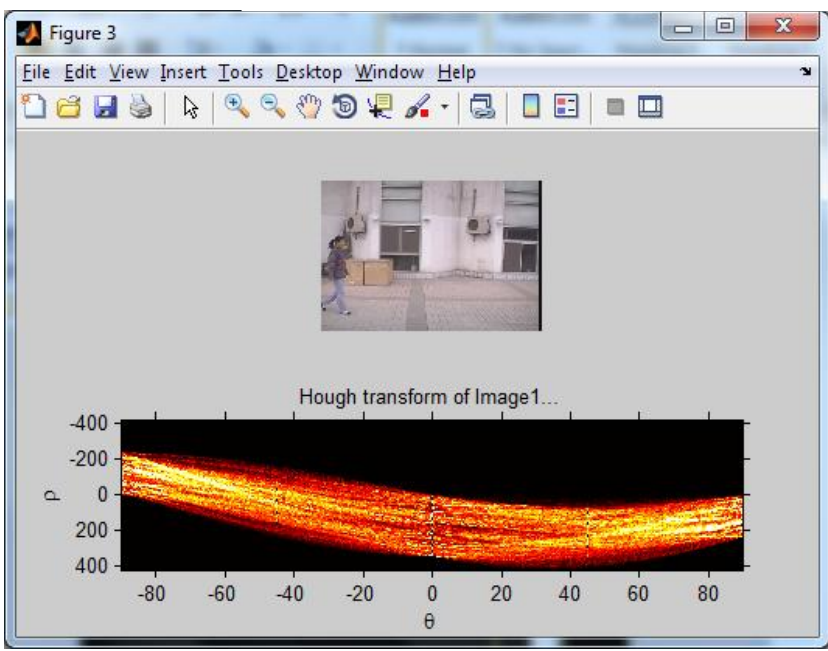

Fig. 6. Feature Extraction (Hough Transform)

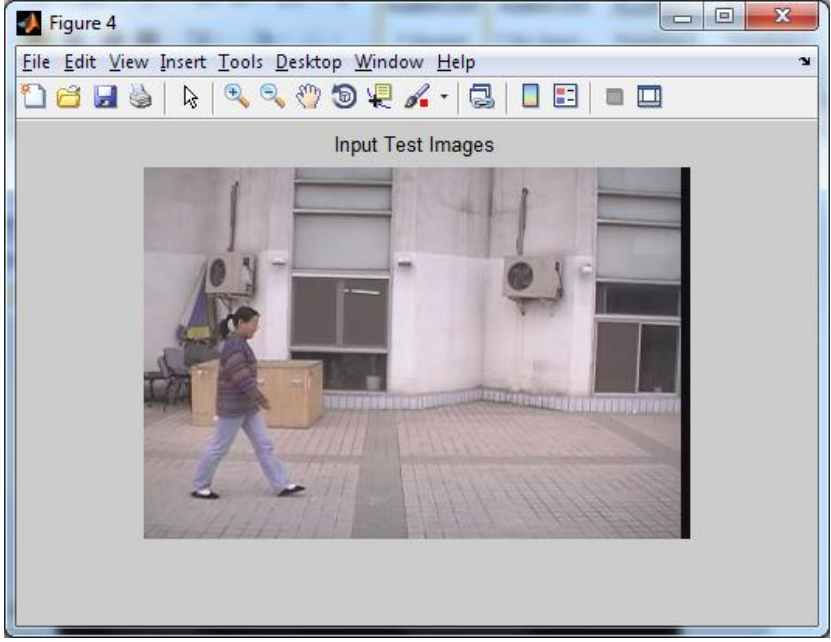

Fig. 7. Test Image Sequence

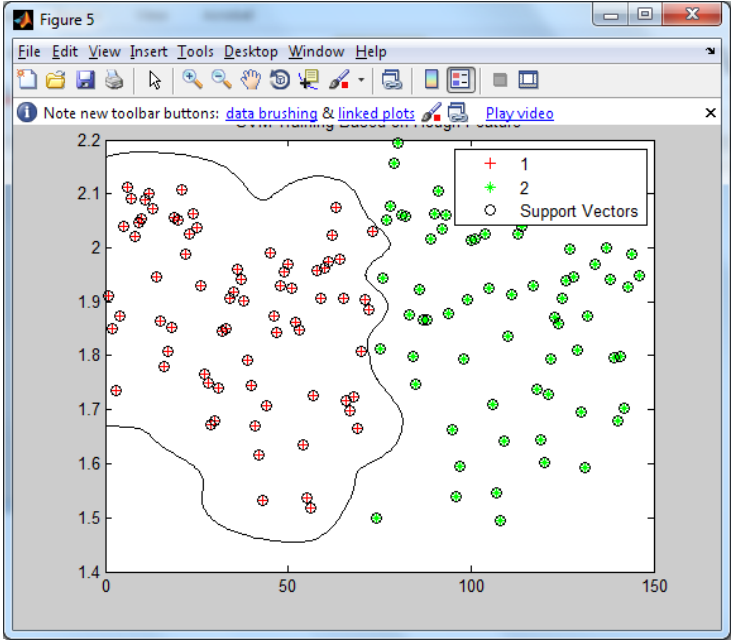

Fig. 8. SVM-Graph 1

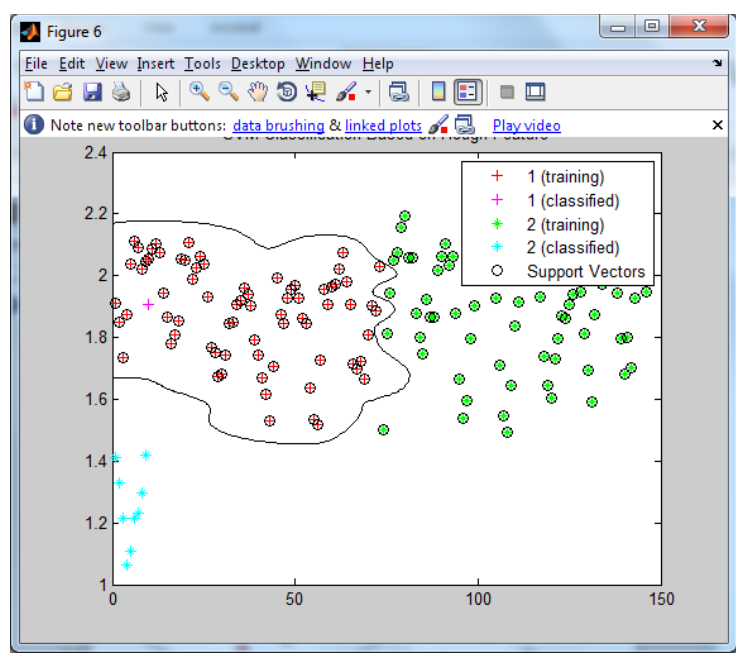

Fig. 9. SVM-Graph 2 


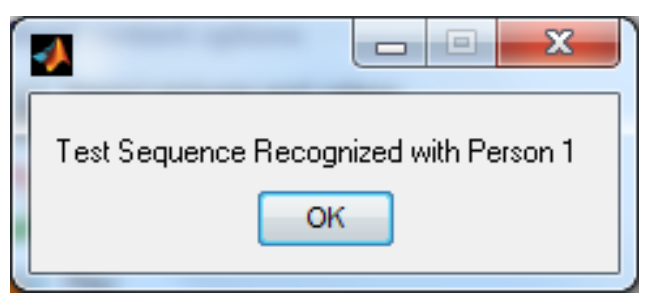

Fig. 10. Person Recognition

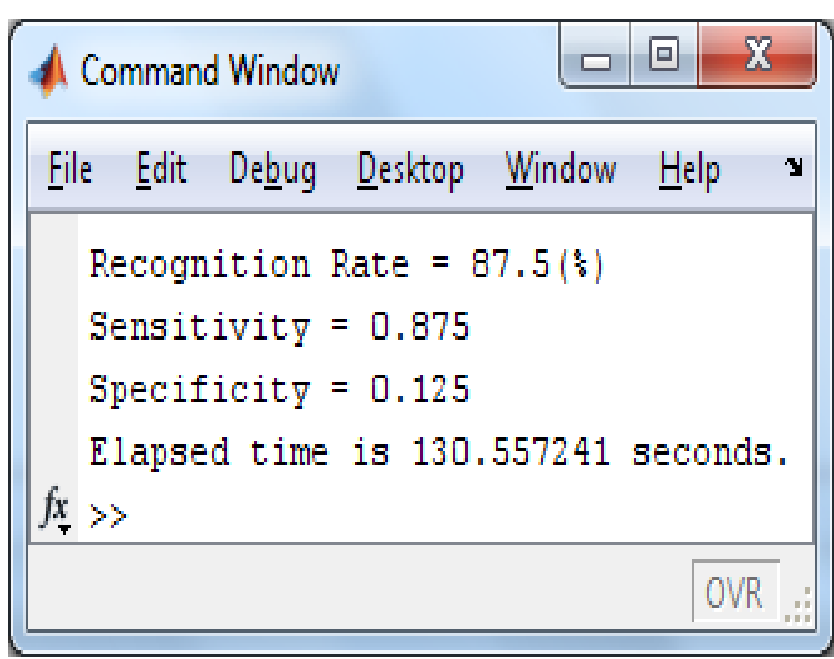

Fig. 11. Recognition Rate and Ellapsed Time

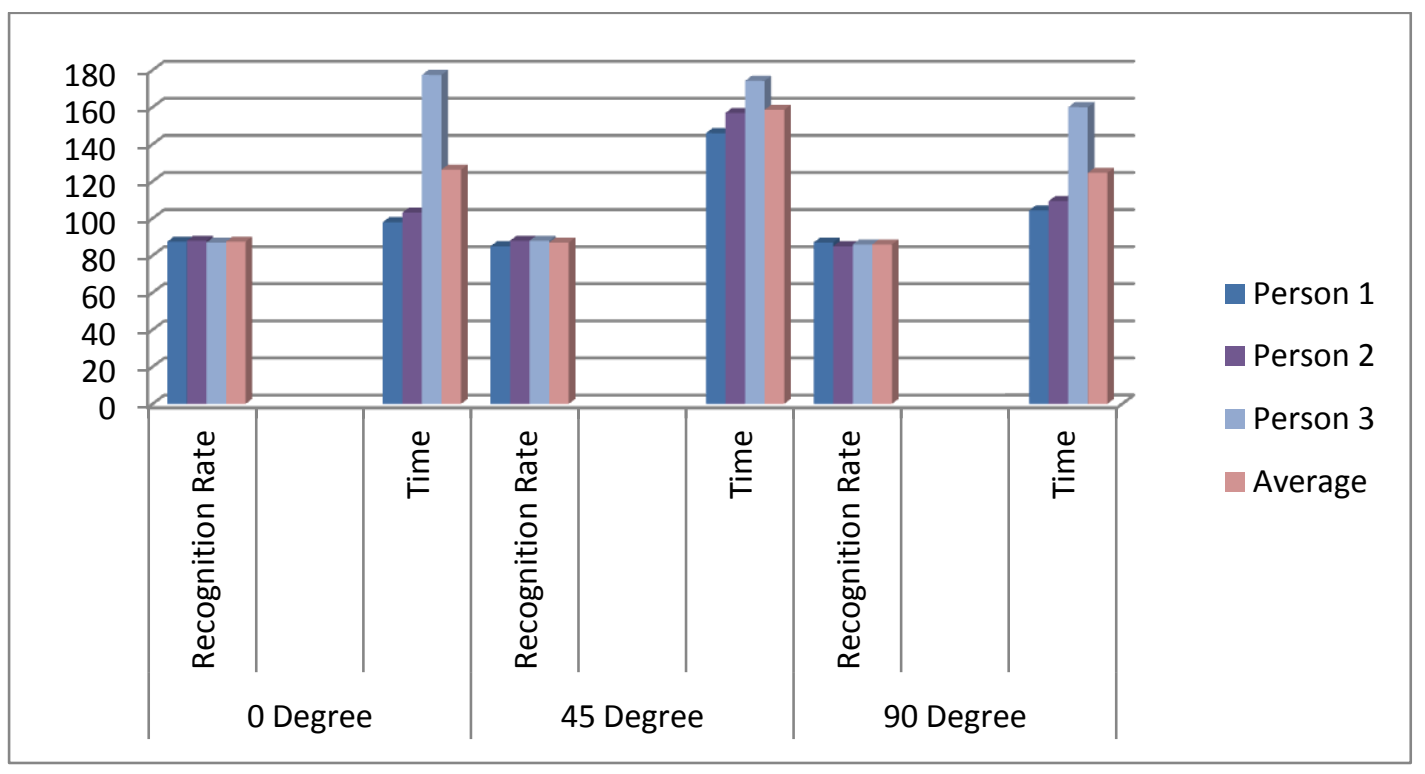

Fig. 13. Graph : Results of Proposed Approach

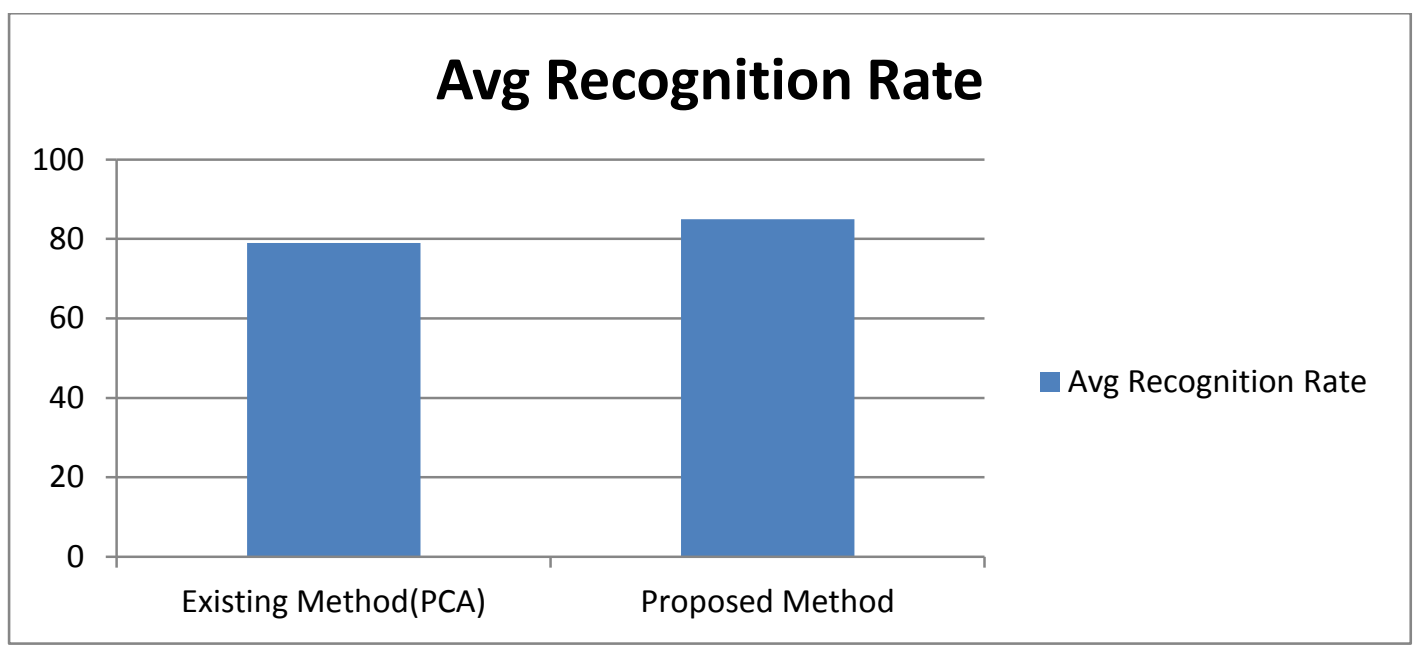

Fig. 13. Graph : Comparision of Existing and Proposed Approach 


\section{CONCLUSION}

In the proposed methodology, person recognition using gait is based on the feature of distance between legs and angle between legs using Hough Transform for the gait sequences. It gives better output result compared to the exiting method. In the proposed method gait can be recognized very well by extracting the features with small variations i.e. different angles etc. Classification is done by using SVM classifier so it gives better performance and output result to recognize the person.

\section{ACKNOWLEDGEMENT}

I would like to express my gratitude to my guide Mr. Mukesh Sakle(Asst. Professor, IT Department), for his great efforts and instructive comments in this work. He has devoted significant amount of his valuable time to organize and discuss the work. He provided me excellent guidance and support. I would like to express my thanks to my dear friend Avani and all my batchmates for their support and help. I would also like to express my thanks to my Parents for their support during this work.

\section{REFERENCES}

[1] Ling-Feng, Wei Jia and Yi-Hai Zhu, "Survey of Gait Recognition,". ICIC 2009, Springer-Verlag Berlin Heideblerg, pp 625-659 (2009).

[2] Junqiu Wang, Yasushi Makihara and Yasushi Yagi, “ Human Tracking and Segmentation supported by Silhouette-based Gait Recognition," 2008 International Conference on Robotics and Automation Pasadena, CA,USA,May 19-23, (2008).

[3] Xuelong Li, Stephen J.Maybank, Shuicheng Yan, Dacheng Tao and Dong $\mathrm{Xu}$, " Gait components and their Application to Gender Recognition," IEEE Trans on
Systems, Man and Cybernetics- part C: Aplications and Review, Vol. 38, no. 2, March (2008).

[4] Zongyi Liu, Laura Malave, Adebola Osuntugun, Preksha Sudhakar and Sudeep Sarkar, "Towards Understanding the limts of Gait Recognition," SPIE International Symposium on Defense and Security: Biometric Technology for Human Identification, April (2004).

[5] Nikolaos V.Boulgouris, Dimitrois Hatzinakos and Konstantinos N. Plataniotis, "Gait Recognition: A Challenging signal processing technology for biometric identification," IEEE Signal Processing Magazine page 78-90 November (2005).

[6] Amit Kale, Aravind Sundaresan, A.N.Rajagopaln, Naresh P.Cuntoor,Amit K. Roy-chowdhury, Volker Kruger and Rama Chellappa, "Identification of Humans Using Gait," IEEE Trans on Image Processing, Vol.13, no.9, September (2004).

[7] Nikolaos V. Boulgouris, "Gait Recognition Using Radon Transform and Linear Discriminant Analysis," IEEE Transactions on Image Processing, Vol. 16, No 3, March (2007).

[8] D. M Tsai and M. F C hen, " Object recognition by linear weight classifier. Pattern Recognition Letters 16, 591 600 (1995)

[9] H.S.Nagendraswamy and D.S.Guru, "A new method of representing and matching two dimensional shapes. International Journal of Image and Graphics," Vol 7, no.2, April (2007).

[10] Mohan Kumar H P and Nagendraswamy H S "Gait Recognition: An Approach Based on Interval Valued Features", IEEE 2013. 